\title{
New air fluorescence detectors employed in the Telescope Array experiment
}

\author{
H. Tokuno ${ }^{\mathrm{a}, *}$, Y. Tameda ${ }^{\mathrm{b}}$, M. Takeda ${ }^{\mathrm{b}}$, K. Kadota ${ }^{\mathrm{c}}$, D. Ikeda $^{\mathrm{b}}$, \\ M. Chikawa ${ }^{\mathrm{d}}$, T. Fujii ${ }^{\mathrm{e}}$, M. Fukushima ${ }^{\mathrm{b}, \mathrm{f}}$, K. Honda $^{\mathrm{g}}$, N. Inoue ${ }^{\mathrm{h}}$, \\ F. Kakimoto ${ }^{\mathrm{a}}$, S. Kawana ${ }^{\mathrm{h}}$, E. Kido ${ }^{\mathrm{b}}$, J. N. Matthews ${ }^{\mathrm{i}}$, T. Nonaka ${ }^{\mathrm{b}}$,

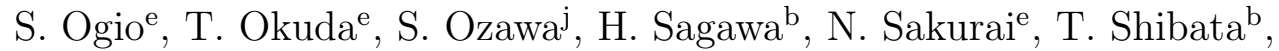

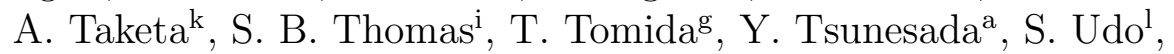 \\ T. Abu-zayyad ${ }^{i}$, R. Aida ${ }^{g}$, M. Allen ${ }^{\mathrm{i}}$, R. Anderson ${ }^{\mathrm{i}}$, R. Azuma ${ }^{\mathrm{a}}$, \\ E. Barcikowski ${ }^{i}$, J.W. Belz ${ }^{i}$, D.R. Bergman ${ }^{i}$, S.A. Blake ${ }^{i}$, R. Cady ${ }^{i}$, \\ B.G. Cheon ${ }^{\mathrm{m}}$, J. Chiba ${ }^{\mathrm{n}}$, E.J. Cho ${ }^{\mathrm{m}}$, W.R. Cho ${ }^{\circ}$, H. Fujii ${ }^{\mathrm{p}}$, T. Fukuda ${ }^{\mathrm{a}}$, \\ D. Gorbunov ${ }^{q}$, W. Hanlon ${ }^{\mathrm{i}}$, K. Hayashi ${ }^{\mathrm{a}}$, Y. Hayashi ${ }^{\mathrm{e}}$, N. Hayashidal, \\ K. Hibino ${ }^{1}$ K. Hiyama ${ }^{\mathrm{b}}$, T. Iguchi ${ }^{\mathrm{a}}$, K. Ikuta ${ }^{\mathrm{g}}$, T. Ishiig , R. Ishimori ${ }^{\mathrm{a}}$, \\ D. Ivanov ${ }^{\mathrm{i}, \mathrm{r}}$, S. Iwamoto ${ }^{\text {, }}$, C.C.H. Jui ${ }^{\mathrm{i}}$, O. Kalashev ${ }^{\mathrm{q}}$, T. Kanbe ${ }^{\mathrm{g}}$, \\ K. Kasahara ${ }^{j}$, H. Kawai ${ }^{\text {, }}$ S. Kawakami ${ }^{\mathrm{e}}$, H.B. Kim ${ }^{\mathrm{m}}$, H.K. Kimº, \\ J.H. Kim ${ }^{\mathrm{m}}$, J.H. Kim ${ }^{\mathrm{t}}$, K. Kitamoto ${ }^{\mathrm{d}}$, K. Kobayashi ${ }^{\mathrm{n}}$, Y. Kobayashi ${ }^{\mathrm{a}}$, \\ Y. Kondo ${ }^{b}$, K. Kuramoto ${ }^{\mathrm{e}}$, V. Kuzmin ${ }^{\mathrm{q}}$, Y.J. Kwon ${ }^{\circ}$, S.I. Lim \\ S. Machida ${ }^{\mathrm{a}}$, K. Martens ${ }^{\mathrm{f}}$, J. Martineau ${ }^{\mathrm{i}}$, T. Matsuda ${ }^{\mathrm{p}}$, T. Matsuura ${ }^{\mathrm{a}}$, \\ T. Matsuyama ${ }^{\mathrm{e}}$, I. Myers ${ }^{\mathrm{i}}$, M. Minamino ${ }^{\mathrm{e}}$, K. Miyata $^{\mathrm{n}}$, H. Miyauchi ${ }^{\mathrm{e}}$, \\ Y. Murano ${ }^{\mathrm{a}}$, T. Nakamura ${ }^{\mathrm{u}}$, S.W. Nam ${ }^{\mathrm{v}}$, M. Ohnishi ${ }^{\mathrm{b}}$, H. Ohoka ${ }^{\mathrm{b}}$, K. Oki ${ }^{\mathrm{b}}$, \\ D. Oku ${ }^{g}$, A. Oshima ${ }^{\mathrm{e}}$, I.H. Park ${ }^{\mathrm{v}}$, M.S. Pshirkov ${ }^{\mathrm{w}}$, D. Rodriguez ${ }^{\mathrm{i}}$, \\ S.Y. Roh ${ }^{t}$, G. Rubtsov ${ }^{\mathrm{q}}$, D. Ryu ${ }^{\mathrm{t}}$, A.L. Sampson ${ }^{\mathrm{i}}$, L.M. Scott ${ }^{\mathrm{r}}$, P.D. Shah ${ }^{\mathrm{i}}$, \\ F. Shibata ${ }^{g}$, H. Shimodaira ${ }^{b}$, B.K. Shin ${ }^{\mathrm{m}}$, J.I. Shino ${ }^{\circ}$ T. Shirahamah ${ }^{\mathrm{h}}$, \\ J.D. Smithi ${ }^{\text {, P. Sokolsky }}{ }^{\mathrm{i}}$, T.J. Sonley ${ }^{\mathrm{i}}$, R.W. Springer ${ }^{\mathrm{i}}$, B.T. Stokes ${ }^{\mathrm{i}}$,

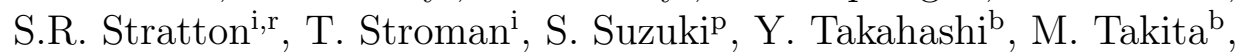 \\ H. Tanakae ${ }^{\mathrm{e}}$ K. Tanaka ${ }^{\mathrm{x}}$, M. Tanaka ${ }^{\mathrm{p}}$, G.B. Thomson ${ }^{\mathrm{i}}$, P. Tinyakover,w, \\ I. Tkachev ${ }^{\mathrm{q}}$, S. Troitsky ${ }^{\mathrm{q}}$, K. Tsutsumi ${ }^{\mathrm{a}}$, Y. Tsuyuguchi ${ }^{\mathrm{g}}$, Y. Uchihori ${ }^{\mathrm{y}}$,

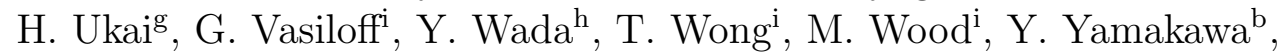

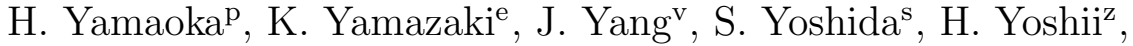 \\ R. Zollinger ${ }^{\mathrm{i}}$, Z. Zundel ${ }^{\mathrm{i}}$ \\ ${ }^{a}$ Tokyo Institute of Technology, Meguro, Tokyo, Japan \\ ${ }^{b}$ Institute for Cosmic Ray Research, University of Tokyo, Kashiwa, Chiba, Japan \\ ${ }^{c}$ Tokyo City University, Setagaya-ku, Tokyo, Japan
}

\footnotetext{
${ }^{*}$ Corresponding author. Tel.: +81-357342462, Fax: +81-357342756. E-mail address: htokuno@cr.phys.titech.ac.jp (H. Tokuno).
} 


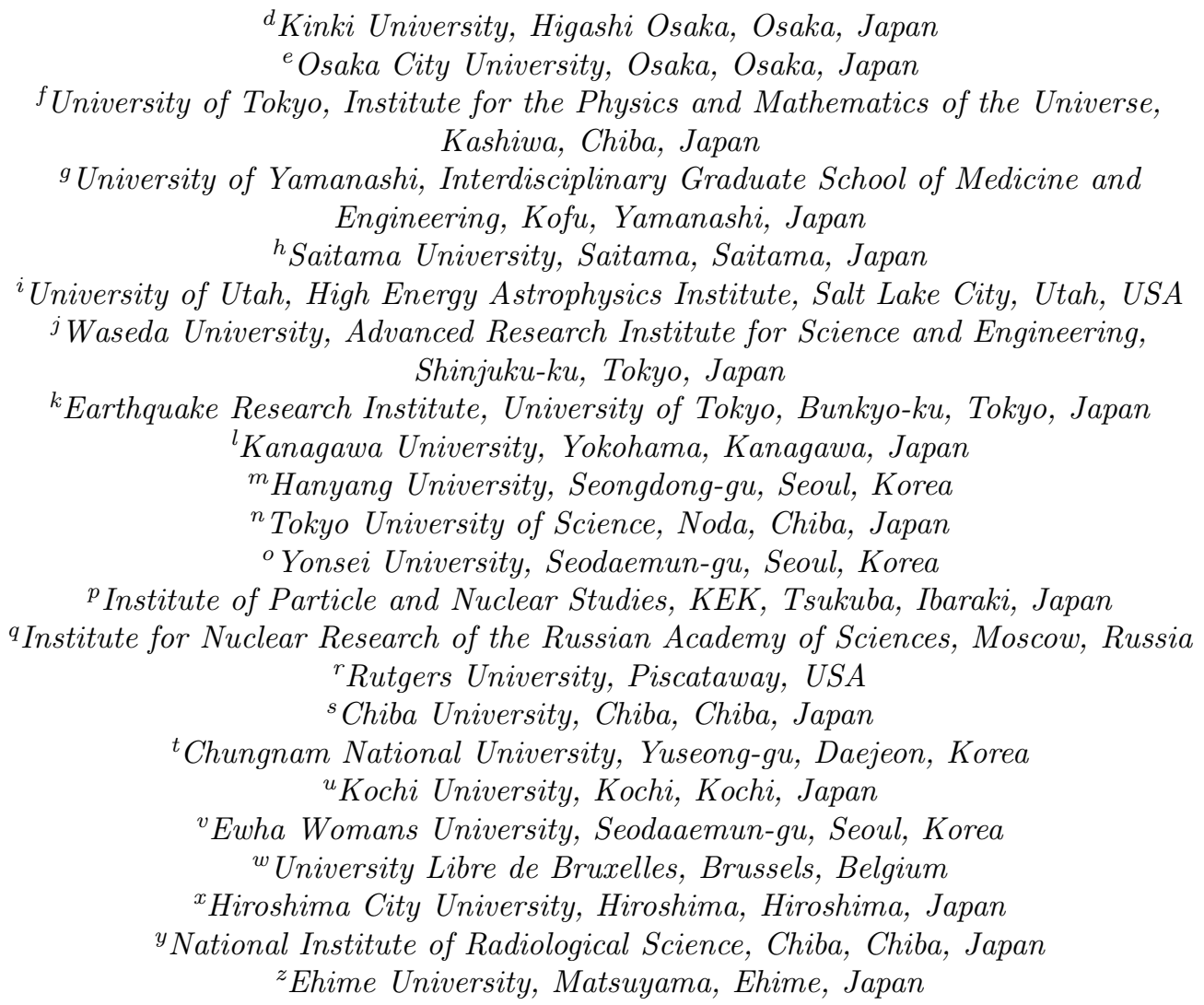

\section{Abstract}

Since 2007, the Telescope Array (TA) experiment, based in Utah, USA, has been observing ultra high energy cosmic rays to understand their origins. The experiment involves a surface detector (SD) array and three fluorescence detector (FD) stations. FD stations, installed surrounding the SD array, measure the air fluorescence light emitted from extensive air showers (EASs) for precise determination of their energies and species. The detectors employed at one of the three FD stations were relocated from the High 
Resolution Fly's Eye experiment. At the other two stations, newly designed detectors were constructed for the TA experiment. An FD consists of a primary mirror and a camera equipped with photomultiplier tubes. To obtain the EAS parameters with high accuracies, understanding the FD optical characteristics is important. In this paper, we report the characteristics and installation of new FDs and the performances of the FD components. The results of the monitored mirror reflectance during the observation time are also described in this report.

Keywords: Ultra high energy cosmic rays, Extensive air showers, Air fluorescence light detectors

\section{Introduction}

2 The Telescope Array project is a collaboration with 120 scientists from 3 four nations (Japan, USA, Korea, and Russia), with the observatory located 4 in Utah, USA [1, 2]. The detectors involved in the Telescope Array experidetector (FD) telescopes. Fig. 1 shows the detector map (squares: SD positions, triangles: FD station positions). The SD array consists of 507 SDs, arranged over an area of approximately $700 \mathrm{~km}^{2}$ with $1.2 \mathrm{~km}$ spacing between the SDs [4]. The SDs measure the arrival timing and particle densities of extensive air showers (EASs) using two-layered plastic scintillators of $3 \mathrm{~m}^{2}$. Three FD stations are placed around the SD array. The FDs measure the air fluorescence light emitted from EASs; the FD data are analyzed and the longitudinal development of EASs is reconstructed to estimate primary energies, arrival directions, and particle species with the aim of studying the 
nature of ultra high energy cosmic rays.

The FD station near the northwest corner of the SD array houses fourteen FDs that were relocated from the High Resolution Fly's Eye (HiRes) experiment (e.g. [3]). For these FDs and for the data obtained using these FDs, we can use the same calibration and analysis methods used in the HiRes experiment [5]. The specifications, configurations, and calibrations of the relocated FDs can be obtained from previous reports on HiRes (e.g. [3]). On the other hand, the southeast (BRM) and southwest (LR) FD sites have twelve FDs each; these FDs were newly designed for the TA experiment. A picture of the FD station is shown in Fig. 2 ,

An FD consists of a primary mirror and a photomultiplier tube (PMT) camera. We employed a spherical mirror to obtain a wide field of view (FOV) with a sufficient focusing power. In addition, the size of the collecting area of the mirror was determined from the maximum distance for detectable EASs at the highest energies. The mirror aperture and curvature radius were determined to be $3300 \mathrm{~mm}$ (area of $6.8 \mathrm{~m}^{2}$ ) and $6067 \mathrm{~mm}$, respectively. This setting realizes the detection of EASs with a sufficiently high accuracy from a distance of $30 \mathrm{~km}$ and with the primary energy of $10^{20} \mathrm{eV}$.

A primary mirror is segmented into eighteen small hexagonal mirrors; the distance between the parallel sides of the hexagonal mirror was $660 \mathrm{~mm}$. A PMT camera consisting of $16 \times 16$ PMTs and having an effective area of $860 \mathrm{~mm} \times 992 \mathrm{~mm}$ is set at a distance of $3000 \mathrm{~mm}$ from the mirror. The FOV of each PMT is approximately $1^{\circ}$, and that of the camera is $15^{\circ}$ in elevation and $18^{\circ}$ in azimuth. Fig. 3 shows a schematic view of the FD telescope frame equipped with a pair of telescopes, one each in the upper and lower 
parts of the frame. The heights of the upper and lower mirror centers and the upper and lower camera centers from the floor are 5.5, 1.5, 6.0, and $2.8 \mathrm{~m}$, respectively. The FOV centers of the upper and lower telescopes are at elevations of $10.5^{\circ}$, and $25.5^{\circ}$, respectively. As shown in Fig. 3, no segment mirror is installed at the center of the primary mirror. At this position, a jig was mounted for alignment of the optical system of the telescope when it was constructed. Similarly, during normal operations, a UV light flasher is mounted here as a standard light source for the calibrations and adjustments of PMT gains [6]. The projected view of the FD station onto the station floor is shown in Fig. 4. The station has six telescope frames; accordingly, the FOV of a station is from $3^{\circ}$ to $33^{\circ}$ in elevation and $108^{\circ}$ in azimuth.

To evaluate the accuracies of the measured shower parameters such as arrival direction, primary energy, and longitudinal development, studies of resolutions and systematic uncertainties of FD telescope optics are important. Thus, we ensured that we accurately manufactured, constructed, and installed the FD telescopes with utmost care and after confirmation of their optical characteristics.

In this paper, we describe the elements that constitute an FD telescope and the installation process of the telescopes. Moreover, the reflectance of segment mirrors, which are continuously monitored after the installations, is also presented. The contents of this paper are as follows. In Sec. 2 and Sec. 3, we report the designs and productions of the PMT cameras and those of the segment mirrors, respectively. Sec. 4 explains the installation of the cameras and mirrors. Mirror reflectance and its variations are described in Sec. 5 . 


\section{Camera production}

In this section, we describe the configuration of the newly designed PMT cameras and the results of user acceptance tests.

\subsection{Photomultiplier tube}

Each camera has 256 PMTs. 7000 PMTs including spares have been produced. The PMT used in the TA experiment, Hamamatsu R9508 based on R6234-01, is specially manufactured for the experiment. The photoelectric surface on the top of a tube is hexagonal in shape with a distance of $60 \mathrm{~mm}$ between the parallel side; its effective area is equivalent to that of circle of $57 \mathrm{~mm}$ diameter [6]. On the bottom of the tube, a printed circuit board of a bleeder and a preamplifier is installed. In order to reduce the contamination of the night sky background photons into fluorescence signals, we use a $4 \mathrm{~mm}$ thick optical filter (SCHOTT AG, BG3). The filter is mounted on the surface of each PMT using a self-fusing tape (Hitachi Chemical Co., Ltd., HIGHBON TAPE No.2) and a polyimide film tape (3M, 5434). The transmittance of BG3 is higher than $80 \%$ in the wavelength range of $305-395 \mathrm{~nm}$, which is within the range of all the major air fluorescence lines [6].

The PMTs were inspected by the supplier before shipping and were selected based on their cathode and anode sensitivities, dark current, and other basic parameters. In addition, the quantum efficiency (including collection efficiency), and cathode uniformity were measured for sampled PMTs, once for 1000 PMTs. We also imposed sufficiently high gains and low noise levels on PMTs at the experimental sites. The minimum required gain was $6 \times 10^{4}$ with the maximum applied high voltage of $-1200 \mathrm{~V}$. 
After installation of camera, we adjusted PMT gains to absolutely calibrated PMTs [7] and measured the uniformity of the PMT response at its photoelectric surface on the sites [6]. During FD observations, each PMT gain was monitored every one hour using UV flashers. We exchanged two of the 6144 PMTs because the gains of these two PMTs had decreased in the first half year. Since then, no PMT has been exchanged.

\subsection{Camera}

A PMT camera has been placed at the primary focus of each FD telescope. Fig. 5 shows the schematic view of the PMT camera. The camera has a UV transparent acrylic window (KURARAY, PARAGLAS-UV00) attached in front of PMTs for protection from dust. The transmittance of the window is more than $90 \%$ in the wavelength range of $300-400 \mathrm{~nm}$ [6]. PMTs were fixed on a $16 \mathrm{~mm}$ thick plate of extra super duralumin and were arranged on a triangular grid with a clearance gap of approximately $1 \mathrm{~mm}$ among them. The gap geometries were measured based on the uniformity of the camera surface response [6].

Signals from the PMTs and the DC power to the PMTs are transferred through shielded LAN cables of category 5 (length: $50-100 \mathrm{~cm}$ ) between the PMTs and the patch panels inside a camera. The patch panels connected to the LAN cables are shown in Fig. 6, We use a shielded 40 core twisted pair cable (Bando Densen Co., Ltd., BIOS-A-2820P) each for sixteen PMTs to transfer the PMT signals from the patch panels in a camera to a data acquisition (DAQ) system in the electronics room with temperature control, situated $20 \mathrm{~m}$ from the camera location. The cable length for the upper and lower cameras is $25.5 \mathrm{~m}$ and $22.3 \mathrm{~m}$, respectively. Detailed descriptions of 
the electronics and DAQ system are presented elsewhere [8, 9].

High voltages $(\mathrm{HVs})$ are applied to PMTs via coaxial cables (Bando Densen Co., Ltd., 1.5D-2V) using an order-made power supply (Takasago Co., Ltd.). The power supply has 256 outputs, and HV value on each output can be controlled and monitored separately through Ethernet, with the accuracy of $\pm 0.2 \%$. This uncertainty corresponds to approximately $\pm 2 \%$ of the PMT gains under our setting. The typical applied HV to PMTs is $-880 \mathrm{~V}$, and the rated maximum voltage is $-1200 \mathrm{~V}$. As a safety measure to protect human beings, PMTs, and the power supply, the power supply is automatically shut down when unusually high values are detected.

A DC power supply (KENWOOD, PW18-3AD) is connected to two patch panels to distribute DC voltage of $\pm 5 \mathrm{~V}$ to the 256 preamplifiers, each connected to one PMT. In this manner, we prepared one DC power supply for each camera.

\section{Segment mirrors and their production}

In this section, we describe the specifications of the segment mirrors employed in the new FD telescopes of the TA experiment, their pre-shipment and acceptance inspections, and the inspection results.

\subsection{Segment mirrors}

The $3.3 \mathrm{~m}$ aperture spherical mirror of the FD telescope is segmented into eighteen small mirrors, as shown in Fig. 3. The mirror is made of borosilicate glass (SCHOTT AG, TEMPAX), and each segment is hexagonal in shape with a thickness of $10.5 \mathrm{~mm}$ and a distance of $660 \mathrm{~mm}$ between the parallel sides. On the surface of the mirror was deposited a $200 \mathrm{~nm}$ thick reflection 
coating of aluminum through vacuum deposition, and on this coating was deposited a $50 \mathrm{~nm}$ thick protective coating of $\mathrm{Al}_{2} \mathrm{O}_{3}$ through anodization process. The wavelength that induces maximum reflectance depends on the thickness of the anodized coating. This thickness was optimized to obtain the maximum reflectance at wavelength of $350 \mathrm{~nm}$.

The optical parameters and fabrication accuracies of segment mirrors depend on the experimental requirement. In order to optimize the optical parameters, we studied the reconstruction accuracies of EASs using raytracing, EAS simulation, and reconstruction programs. From our studies, we determined the specifications of the optical parameters and the fabrication accuracies; the curvature radius of the segment mirror was $6067 \mathrm{~mm}$ $\pm 100 \mathrm{~mm}$, the spot size at the focal point was smaller than $40 \mathrm{~mm}$ (it is comparable size of the PMT surface), and the reflectance in the wavelength range of $300 \mathrm{~nm}-400 \mathrm{~nm}$ was more than $85 \%$.

\subsection{Segment mirror production}

Segment mirrors were manufactured (Sanko Seikohjyo Co., Ltd.) from January to November, 2004, for the BRM station and from March to December, 2006, for the LR station. In all, 500 segment mirrors, including spares, were produced. The procedure for mirror production is as follows. First, the segment mirror was shaped by heating a planar glass on a ceramic model plate in a temperature-controlled electric oven. After this spherical mirror fabrication, the mirrors were selected through curvature radius measurement. Next, the mirror surface was coated with $200 \mathrm{~nm}$ thick aluminum through the vacuum deposition. To protect the surface, $50 \mathrm{~nm}$ thick $\mathrm{Al}_{2} \mathrm{O}_{3}$ crystal layer was coated on it; these $\mathrm{Al}_{2} \mathrm{O}_{3}$ crystals were produced from a 
solution containing ammonium hydroxide, tartaric acid, and ethylene glycol. After the surface fabrication, the mirrors were selected based on reflectance measurement. Finally, a $150 \mathrm{~mm}$ diameter support disk made of the same material as the mirror and a stainless steel flange were bonded to the back of the mirror using glue (3M, Dymax 840). The flange was used to support the segment mirror from the telescope frame.

\subsection{Acceptance test for curvature radius and spot size}

For the acceptance inspections, we measured the curvature radius and spot size at the curvature center. From our ray-tracing studies, we estimated an acceptable spot diameter of $20 \mathrm{~mm}$, in which $90 \%$ of the reflected photons fall, at the center of the curvature. Because of this phenomenon, parallel incident light makes a spot of $40 \mathrm{~mm}$ diameter on the camera, in which $68 \%$ of reflected photons fall, in the case of a normal FD observation. We developed a test system to measure the curvature radius of mirrors as the distance corresponding to the minimum spot size. Fig. 7 shows the schematic view of the system, and Fig. 8 shows the photograph of the same. This system consisted of a linear precision motion stage (range: $\pm 250 \mathrm{~mm}$, accuracy: $0.04 \mathrm{~mm}$, Chuo Precision Industrial Co.,Ltd., MM STAGE ALS-250-C2P with controller QT-CD1); a diffused light source, i.e., a green laser (Kochi Toyonaka Giken Co.,Ltd., GLM-D2) with a diffuser plate; an image scanner (range: 16 bit, resolution: 2400-4800 dpi, Canon, CanoScan LiDE80); and a laser distance meter (accuracy: $\pm 1 \mathrm{~mm}$, Murakami Giken, DIST pro ${ }^{4} \mathrm{a}$ ). The diffused light source was set $100 \mathrm{~mm}$ away from the optical axis of the mirror, and the center of the scanner's sensitive area was fixed at the axisymmetrical point to the optical axis. Both the light source and the scanner were mounted 
on the motion stage and were collectively moved between $6067 \pm 100 \mathrm{~mm}$ from the center of the mirror in $5 \mathrm{~mm}$ steps. Next, we determined the curvature radius by searching for the point where the spot size was minimized. From our ray-tracing calculations, we confirmed that the spot size of the light reflected on the scanner was minimized at the same distance as the curvature radius in spite of the off-axis alignments of the light source and the scanner.

A typical observed spot image is shown in Fig. 9. In this analysis, the image size was defined as the diameter of the circle in which $90 \%$ of the detected photons fall; this circle was centered at the weighted center of the image. Circles in Fig. 10 show an example relation between the spot size and the distance from a mirror. From the data points (circles) in Fig. 10, we obtain the curvature radius to be $6082 \mathrm{~mm}$. Typically, as shown in Fig. 9, the image shape was non ideal circle, because the surface of the mirrors was slightly elliptical. Accordingly, we required an additional criterion for elliptical mirrors. We also measured spot sizes of elliptical mirrors as follows. First, we fitted a shot image at the beginning of a distance of $5967 \mathrm{~mm}$ with a line (called $\mathrm{X}$ axis), and another line (called $\mathrm{Y}$ axis) was obtained perpendicular to the $\mathrm{X}$ axis. These axes were fixed during the subsequent calculations. The shot image was projected on these axes, and the projected spot sizes on these axes were calculated at each distance step that the stage away from the mirror. Finally, we obtained the curvature radii on these axes from the minimum of these plots. We also required the curvature radii on the two axes to be within $6067 \pm 100 \mathrm{~mm}$. The projected spot sizes on the $\mathrm{X}$ and $\mathrm{Y}$ axes for the sampled mirror are shown as square and triangle points, respectively, in Fig. 10. From this figure, the curvature radii on the $\mathrm{X}$ and $\mathrm{Y}$ 
axes are $6072 \mathrm{~mm}$ and $6087 \mathrm{~mm}$, respectively, and these radii also satisfy the above requirement. Fig. 11 shows the distribution of the curvature radius of the accepted mirrors, and Fig. 12 shows the distribution of the normal spot size in diameter at the curvature radius. The curvature radius of the accepted mirrors is $6057 \mathrm{~mm}(1 \sigma:-20 /+30 \mathrm{~mm})$, and their spot size is $12 \mathrm{~mm}(1 \sigma$ : $-2 /+3 \mathrm{~mm})$ in diameter. Henceforth, $1 \sigma$ indicates $68 \%$ confidence level.

From the acceptance test, we found that $5 \%$ mirrors were unacceptable. To complement the required number of mirrors, other acceptable mirrors were produced additionally.

\subsection{Acceptance test for reflectance}

We employed two different acceptance tests for the segment mirror reflectance. One is an accurate measurement in the laboratory for a wide wavelength range for sampled mirrors, which was performed by the manufacturer. The other is a simple measurement with a portable spectrophotometer for a narrow wavelength range for all the segment mirrors.

As it is difficult to measure the reflectance of the curved and large segment mirrors accurately, the manufacturer produced small flat pieces of mirrors simultaneously with sampled segment mirrors and precisely measured the reflectance of the flat pieces as part of the delivery inspection process. The reflectance of the small flat mirrors was measured using a spectrometer (Jasco Inc., Ubest V-550), whose range, resolution, and accuracy are 190-900 nm, $2 \mathrm{~nm}$, and 1\%, respectively. Fig. 13 shows the typical reflectance of sampled small flat mirrors. This reflectance is more than $90 \%$ in the wavelength range of 300-400 nm, containing all the major air fluorescence lines.

We use a portable spectrophotometer (Konica Minolta, Inc., CM-2500d) 
for acceptance inspections of all the manufactured segment mirrors and for routine monitoring of reflectance. The range and its resolution are $360-740 \mathrm{~nm}$ and $10 \mathrm{~nm}$, respectively. The nominal uncertainties of measurement are less than $1 \%$. To confirm the accuracy and stability of the spectrophotometer, we measured the reflectance of a reference mirror eighteen times in the period from June 2008 to November 2010. The reference mirror (Ocean Optics Inc., STAN-SSH-NIST) is quartz-coated; its reflectance was measured by the National Institute of Standards and Technology (NIST), USA. Fig. 14 shows a comparison between our measurements and those done by NIST. From this figure, the systematic differences are within $\pm 1 \%$ and the deviations are within $\pm 1 \%$ in the concerned wavelength range. Fig. 15 shows the variation in the reflectance of the reference mirror at the wavelength of $360 \mathrm{~nm}$, measured using a portable spectrophotometer. The stability was $0.6 \%(1 \sigma)$ during the period from June 2008 to November 2010.

The typical reflectance of a primary mirror composed of eighteen segments is shown in Fig. 16. This figure shows that the reflectance in the wavelength range of 360-400 $\mathrm{nm}$ is higher than $90 \%$. The distribution of the reflectance at $360 \mathrm{~nm}$ for all the segment mirrors is shown in Fig. 17, This distribution shows that the differences in the reflectance depend on 108 sampling points of each primary mirror and that the variance is less than $1 \%$, which is within measurement accuracies. Fig. 18 shows the reflectance of the primary mirrors in BRM and LR stations, which is the average of eighteen segment mirrors, with $1 \sigma$ error bars. All the telescopes fulfilled the requirement that the averaged reflectance was greater than $90 \%$ in the wavelength range of $360 \mathrm{~nm}$. 


\section{Telescope installation}

Mirrors and cameras were shipped from Japan to our observatory in Utah, USA, and these items were installed at BRM from February 2005 to July 2006 and at LR in March 2007. In this section, we describe the installations of the FD telescopes and their alignment.

\subsection{Installation of telescope frame}

As shown in Fig. 4, six telescope frames were installed in each station (BRM and LR) with each frame having two telescopes. Hence, in all, twelve telescopes were installed in each station.

A station building has three rolling doors to protect the telescopes from sunlight, rain, wind, and other natural conditions. The telescopes are directly exposed to air during FD observation, because no other protection windows were installed.

When the wind speed was higher than $15 \mathrm{~m} / \mathrm{s}$, doors were closed to prevent themselves from being stuck. We required less than $0.1^{\circ} \mathrm{FOV}$ deflections of the telescope with wind speeds lower than $15 \mathrm{~m} / \mathrm{s}$. Wind-induced deflections of the telescopes were studied by the manufacturer (Mitsui Engineering \& Shipbuilding Co., Ltd.) using simulation programs and by applying windtunnel tests on a structure model of a station including telescopes. From their studies, winds from the side of the telescope building induce the maximum deflections in the telescope frame. Because winds pass through the building from one door to another, their effective speed is not reduced by the building. However, the current design of the frame fulfills our requirement with regard to frame deflections, even in the worst condition. Based 
on the studies of the manufactures, the design of telescope frame structure was fixed.

To install the telescope frames precisely, we conducted a survey and drew datum lines and points for telescope geometry. Using a gyro-compass, the absolute azimuthal direction was measured with reference to a mountain's peak. Uncertainties of the gyro-compass measurement was $1^{\prime}$, which was confirmed from the star observation using the gyro-compass. Three datum lines, shown as solid lines in Fig. [4 were drawn on the floor with relative accuracies of $1^{\prime}$ between these lines. Azimuthal lines of each telescope frame, shown as dashed lines in Fig. 4, were drawn from the datum lines with accuracies of $0.1^{\circ}$ using a measuring tape. From the azimuthal lines, reference points for the telescope frames were marked with accuracies of $1 \mathrm{~mm}$.

We installed the telescope frames, which can be separated into frames for cameras and mirrors, as shown in Fig. 3 , in accordance with the following procedures. The telescope frames were placed and fixed on the reference points, and before installing real cameras, a prototype standard camera was attached to the camera frames. This prototype standard camera had the same weight and size of a real camera and was equipped with reference lines on the surface for adjusting positions. A steel cylinder with a diameter of $500 \mathrm{~mm}$ is mounted at the center of each primary mirror, as shown in Fig. 3. The central cylinder was used to adjust the alignment of segment mirrors and the camera of the telescope.

Telescope directions were adjusted by the following procedures. First, we installed a laser range meter on the central cylinder. Next, we adjusted the elevation angle to the specified value, monitored using a digital tilt meter, 
(Digital Protractor, Pro 360), with an accuracy of $\pm 0.2^{\circ}$. We adjusted the azimuthal angle of the cylinder for the central axis laser to point the string of a plumb $3000 \mathrm{~mm}$ away from the cylinder set exactly above the reference line on the floor. Then, the position of the camera frame was precisely adjusted with reference to the central axis laser. The upper limit for the installed error on the distance between the camera and the cylinder is $\pm 30 \mathrm{~mm}$. However, this misalignment was resolved when we installed mounts of segment mirrors whose heights were adjusted to remove the distance error. On the other hand, to reduce the construction cost, we required relatively low accuracies of the direction of the camera, which are within $1^{\circ}$. This affects the negligible uncertainties of the effective area of the camera, the order of which is $\cos \left(1^{\circ}\right)$. After the adjustment of the camera position, the prototype standard camera was unmounted from the camera frame.

Finally, the positions of the segment mirror mount were adjusted. Fig. 19 shows the schematic view of a jig called "BANANA1" for adjusting the positions of the segment mirror mount. BANANA1 was mounted on the central cylinder of mirrors and can be rotated around the central axis of the cylinder. The equipment had two arms, one of which had three protruding legs of different lengths; the other arm had a counter weight. By rotating BANANA1, we adjusted the length of every segment mirror mount for one of the three legs on BANANA1 to be aligned with the reference point on the mount surface. At the same time, the direction of each segment mirror was roughly adjusted using BANANA1. 


\subsection{Installation of cameras and mirrors}

The cameras and mirrors were set on the telescope frame by the following procedures. We set the segment mirrors on their mounts and precisely aligned each direction by screwing in and out two adjusting bolts on a mount. To adjust the direction of segment mirrors, we use an equipment called "BANANA3", shown in Fig. 20, The equipment was mounted on the camera frame, and a white board was perpendicularly set on the optical axis of the primary mirror. The distance between segment mirrors and the board was $6067 \mathrm{~mm}$, which is the same distance as the curvature radius of the mirrors. Green LEDs were connected to the white board, 5, 10, and $15 \mathrm{~cm}$ away from the center of curvature, as shown in Fig. 21. When the direction of the segment mirror was adjusted, the LED lights were reflected to the symmetrical point about the center of curvature. The direction was adjusted with an accuracy of less than $0.1^{\circ}$, which was estimated from the accuracy of center position determination of reflected light of $5 \mathrm{~mm}(1 / 4$ diameter of the reflected lights) and from the distance between mirror and the white board, $6067 \mathrm{~mm}$. For parallel lights, this setting accuracy provided us the spots of $40 \mathrm{~mm}$ diameter, in which $68 \%$ of reflected photons fall, at the camera surface. After these adjustments, the segment mirror mounts were locked up.

PMT cameras were mounted on the camera frames with an accuracy of $10 \mathrm{~mm}$ on the camera plane. This accuracy corresponds to $\sim 0.2^{\circ}$ uncertainty of the telescope's pointing direction. PMT cameras were connected by signal, HV, and DC cables. Finally, the wiring was confirmed through test operations. 
In all, the directions of the telescope are adjusted with a few $0.1^{\circ}$ accuracies. These directions were confirmed with a star light analysis, which uses a similar method as in reference [10]. Our FDs record air fluorescence signals with background photons (BGPs), as we employ a DC coupling for the PMT signal readout. Thus, we obtain the variations in BGP with a normal trigger rate of $2-3 \mathrm{~Hz}$. When a star traverses the FOV of a PMT, the number of BGPs increases and decreases in a few minutes. The time variations of BGPs are simulated from star positions and telescope directions. Using these estimated time variations of BGPs, we studied the telescope directions and spot sizes of the primary mirror. The correction values of the FOV direction of FD obtained from this study are used in our simulation and the EAS reconstruction programs. Our results of the star light analysis will be reported in a future paper.

\section{Mirror reflectance monitoring}

The reflectance of the segment mirrors is monitored every months. Mirror reflectance decreases with time, because our FDs are exposed to the air directly and because dust is attached to the mirrors during observations. In addition, the reflectance of lower mirrors decreases more rapidly than that of upper mirrors. Fig. 22 shows the time variations of the averaged mirror reflectance at $360 \mathrm{~nm}$ of a typical lower telescope LR04. The largest decrement of $4 \%$ occurred between June 2007 and March 2008. When we analyze the FD data from that period, we take this $4 \%$ as the systematic uncertainty of the mirror reflectance.

Every summer, the mirrors are washed using purified water and deter- 
gent for sensitive equipments (Alconox, Inc., LIQUI-NOX). Purified water is obtained from tap water filtered through charcoal and deionization filters. After washing, the mirrors are rinsed using purified water and dried naturally. Fig. 22 shows that the reflectance of the mirror after washing is recovered to the same level as in the first installation. Fig. 23 shows the differences in mirror reflectance before and after washing at each wavelength; these differences have no clear wavelength dependencies. Based on this result, we mainly monitor the reflectance at $360 \mathrm{~nm}$.

\section{Summary}

In Japan, we have produced PMT cameras and mirrors for use in the air fluorescence detector of the Telescope Array experiment. The cameras and mirrors that passed our acceptance inspections were shipped to our experimental site in Utah, USA. The telescope frames, mirrors, and cameras were installed at the FD stations. The directions of the telescope are adjusted with an accuracy of a few $0.1^{\circ}$, and the directions are confirmed with a star light analysis. These parameters are used in our detector simulation and the EAS reconstruction programs. Monitoring of the performances of the cameras and mirrors begin according to the following schedules: the gains of PMT camera, hour by hour during FD operation; mirror reflectance, every few months. Our mirrors are washed every summer, and their reflectance after washing is recovered to the same level as in the first installation. 


\section{Acknowledgments}

The Telescope Array experiment is supported by the Japan Society for the Promotion of Science through Grants-in-Aid for Scientific Research on Specially Promoted Research (21000002) "Extreme Phenomena in the Universe Explored by Highest Energy Cosmic Rays", and the Inter-University Research Program of the Institute for Cosmic Ray Research; by the U.S. National Science Foundation awards PHY-0307098, PHY-0601915, PHY0703893, PHY-0758342, and PHY-0848320 (Utah) and PHY-0649681 (Rutgers); by the National Research Foundation of Korea (2006-0050031, 20070056005, 2007-0093860, 2010-0011378, 2010-0028071, R32-10130); by the Russian Academy of Sciences, RFBR grants 10-02-01406a and 11-02-01528a (INR), IISN project No. 4.4509.10 and Belgian Science Policy under IUAP VI/11 (ULB). The foundations of Dr. Ezekiel R. and Edna Wattis Dumke, Willard L. Eccles and the George S. and Dolores Dore Eccles all helped with generous donations. The State of Utah supported the project through its Economic Development Board, and the University of Utah through the Office of the Vice President for Research. The experimental site became available through the cooperation of the Utah School and Institutional Trust Lands Administration (SITLA), U.S. Bureau of Land Management and the U.S. Air Force. We also wish to thank the people and the officials of Millard County, Utah, for their steadfast and warm support. We gratefully acknowledge the contributions from the technical staffs of our home institutions and the University of Utah Center for High Performance Computing (CHPC). We thank HAMAMATSU Photonics K.K., Sanko Seikohjyo Co., Ltd., Quality Steel Fabricating And Welding, Inc, T\&D Maintenance, Nitto Kogyo Cor- 
429

poration, and Mitsui Engineering \& Shipbuilding Co., Ltd. for their kind support.

\section{References}

[1] H. Kawai et al., Nucl. Phys. B Proc. Suppl, 175-176, (2008) 221

[2] H. Kawai et al., J. Phys. Soc. Jpn. Suppl. A, 78 (2009) 108

[3] T. Abu-Zayyad et al., NIM A, 450 (2000) 253

[4] T. Nonaka et al., Proc. of 30th ICRC, 5 (2007) 1005, and now preparing a detailed paper

[5] J. N. Matthews et al., Proc. of 30th ICRC, 5 (2007) 1157

[6] H. Tokuno et al., NIM A, 601 (2009) 364

[7] H. Tokuno et al., Proc. of 30th ICRC, 5 (2007) 1013, and now preparing a detailed paper

[8] Y. Tameda et al., NIM A, 609 (2009) 227

[9] A. Taketa et al., Proc. of 29th ICRC, 8 (2005) 209, and now preparing a detailed paper

[10] P. A. Sadowski et al., Astropart. Phys, 18 (2002) 237 


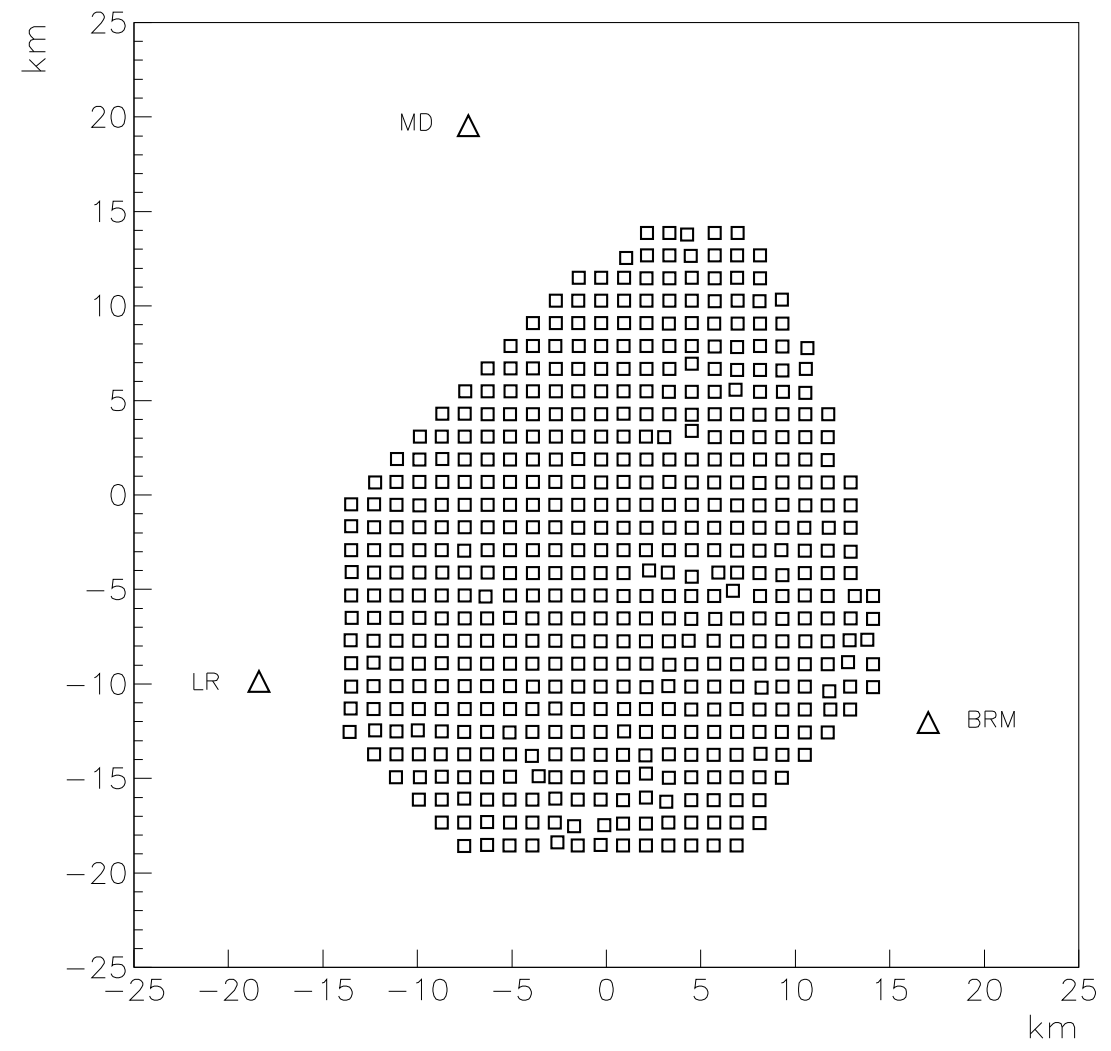

Figure 1: Detector positions in the telescope array experiment. Squares: surface detectors (SDs), triangles: fluorescence detector (FD) telescope stations. SD array area: $700 \mathrm{~km}$, SD spacing: $1.2 \mathrm{~km}$, and distance between FD stations: $\sim 35 \mathrm{~km}$. 


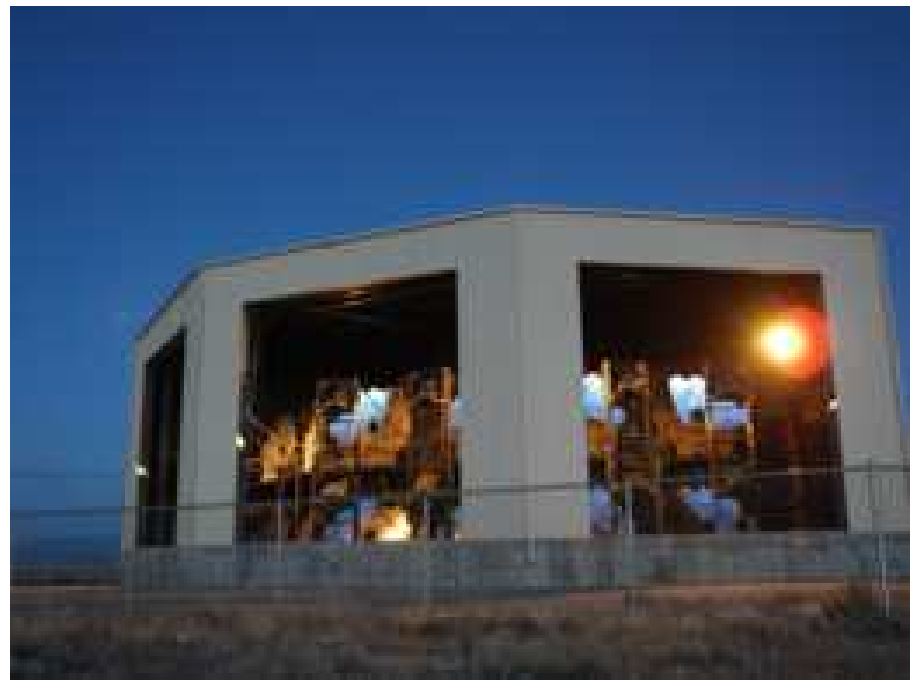

Figure 2: Picture of the fluorescence detector station. 


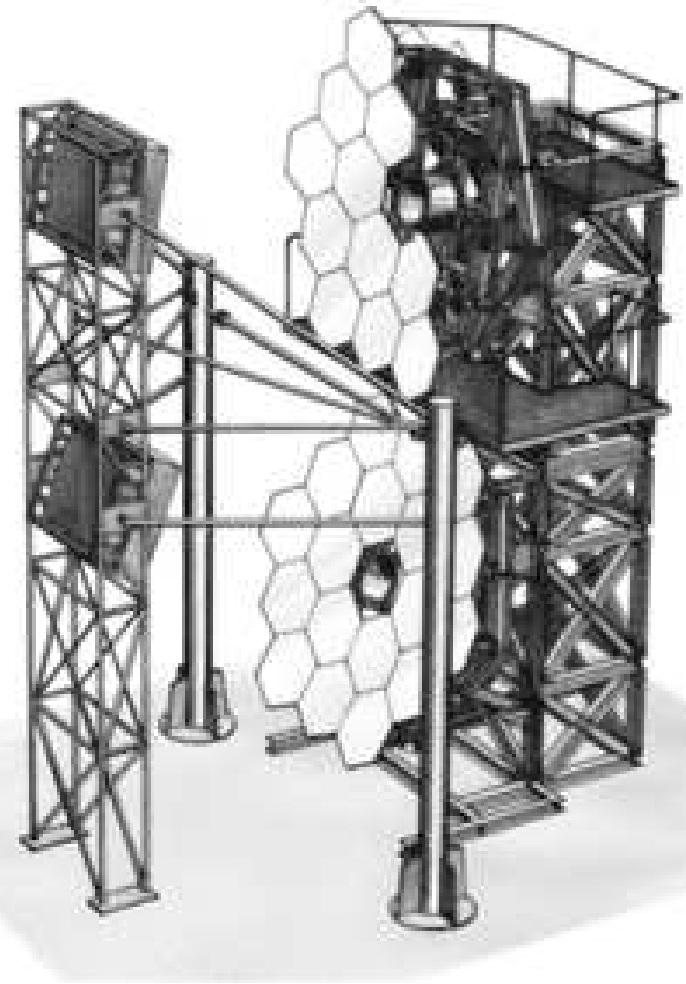

Figure 3: Schematic view of the air fluorescence detectors. For visibility of mirror mounts, seven segment mirrors are removed from the upper telescope. 


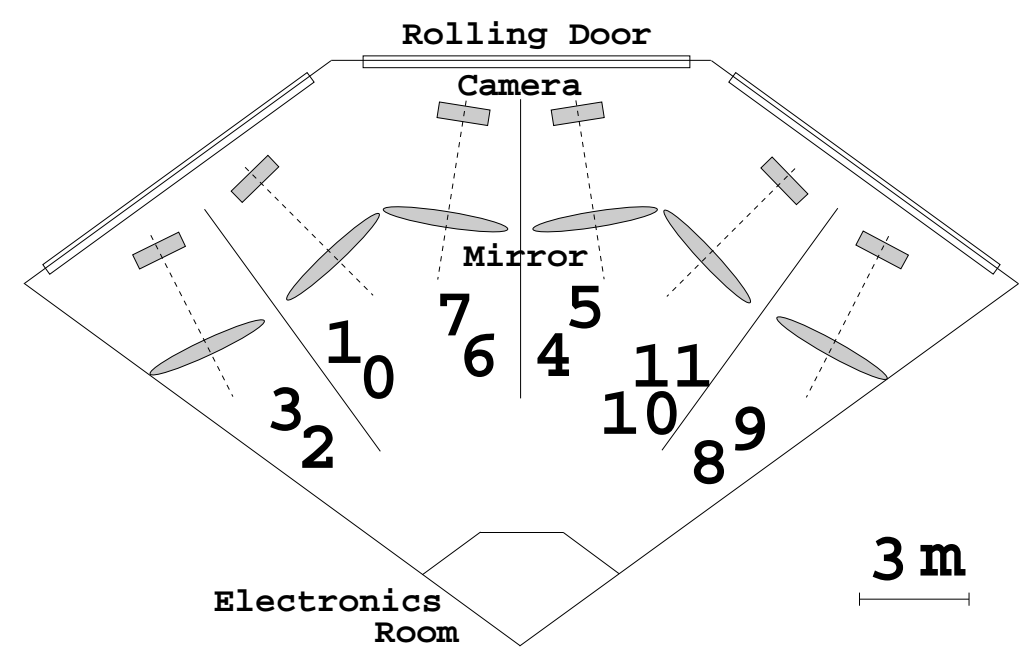

Figure 4: Schematic projected view of the FD station onto the floor (solid lines: datum lines, dashed lines: azimuthal lines of each telescope frame). The telescopes are numbered according to their FOVs, as shown in this figure; the lower (upper) telescopes have even (odd) numbers. 


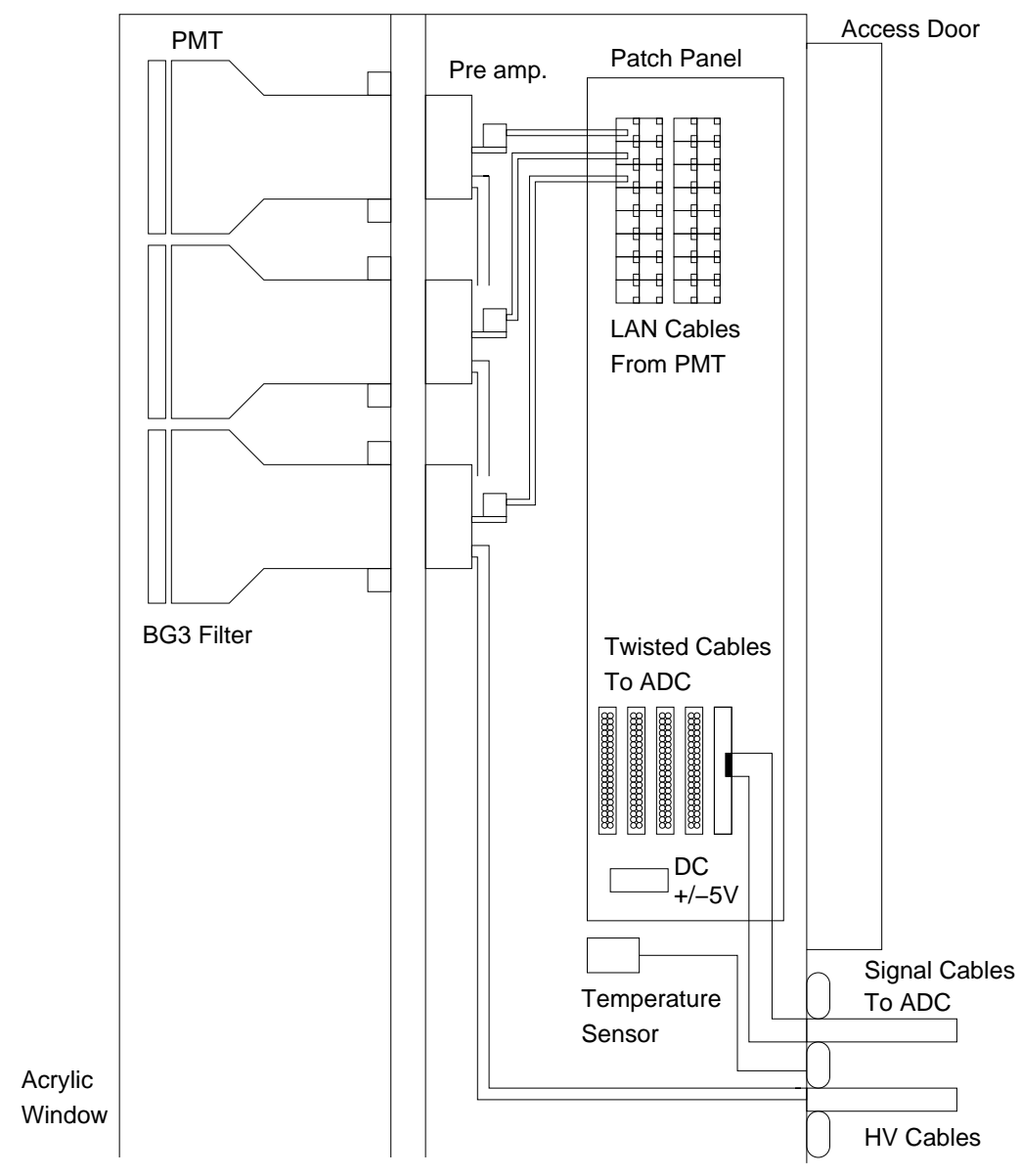

Figure 5: Schematic side view of a PMT camera. Inside the camera, there are 256 PMTs, connected with signal and HV cables, and a temperature sensor. 


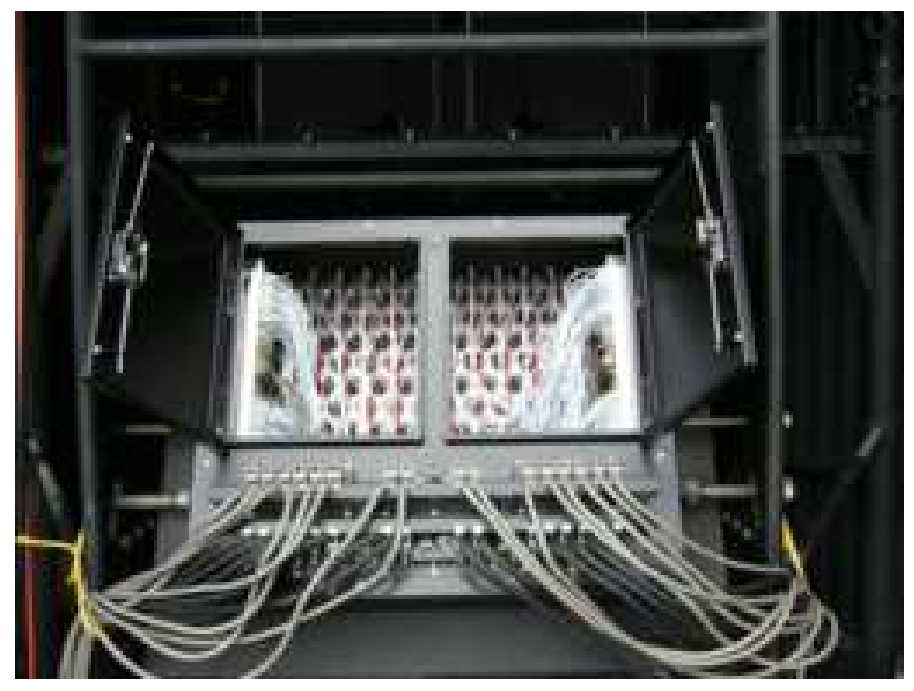

Figure 6: Rear view of the PMT camera. 


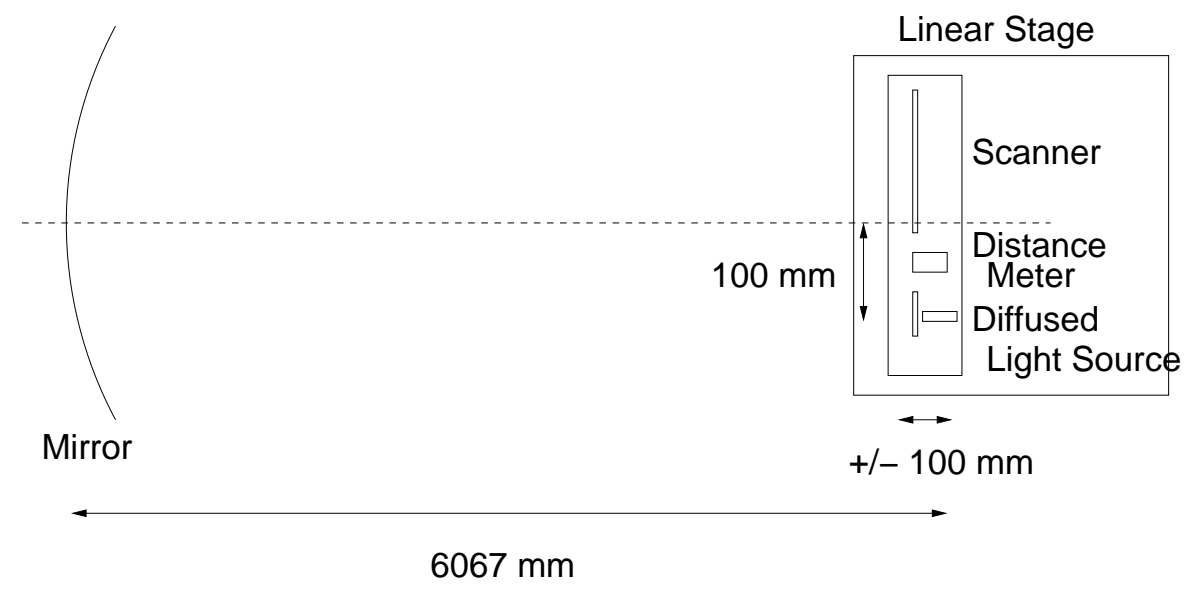

Figure 7: Setup for the measurement of curvature radius of segment mirror. 


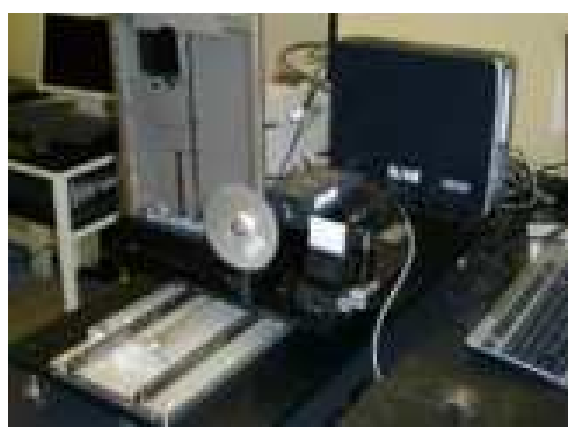

Figure 8: Photograph of the equipment for measuring the curvature radius of segment mirror. This equipment includes a scanner, a distance meter, and a laser source with a diffuser plate, placed to the right on the linear stage. 


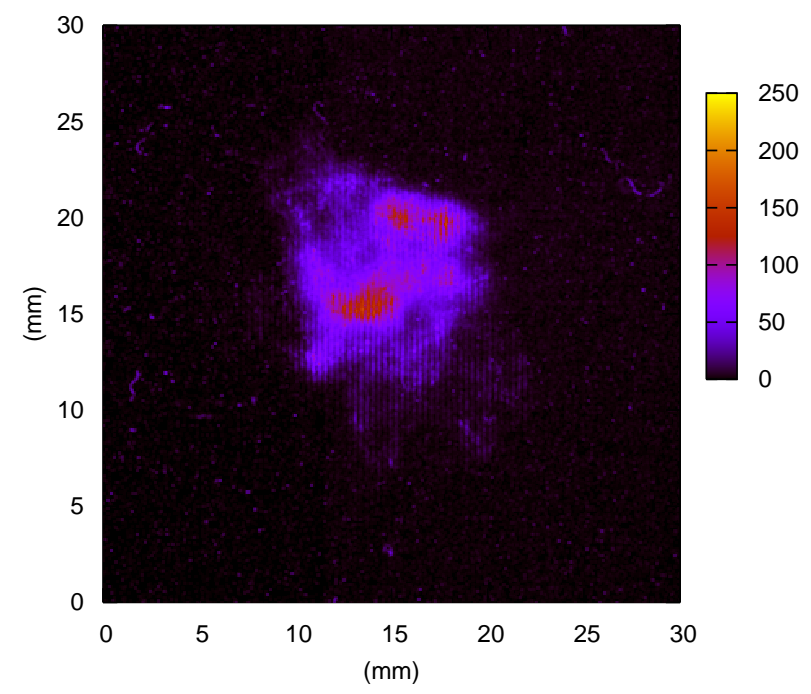

Figure 9: Scanned image of reflected light at the distance equal to the curvature radius of the mirror. Colors show intensities of light in the electrical article. 


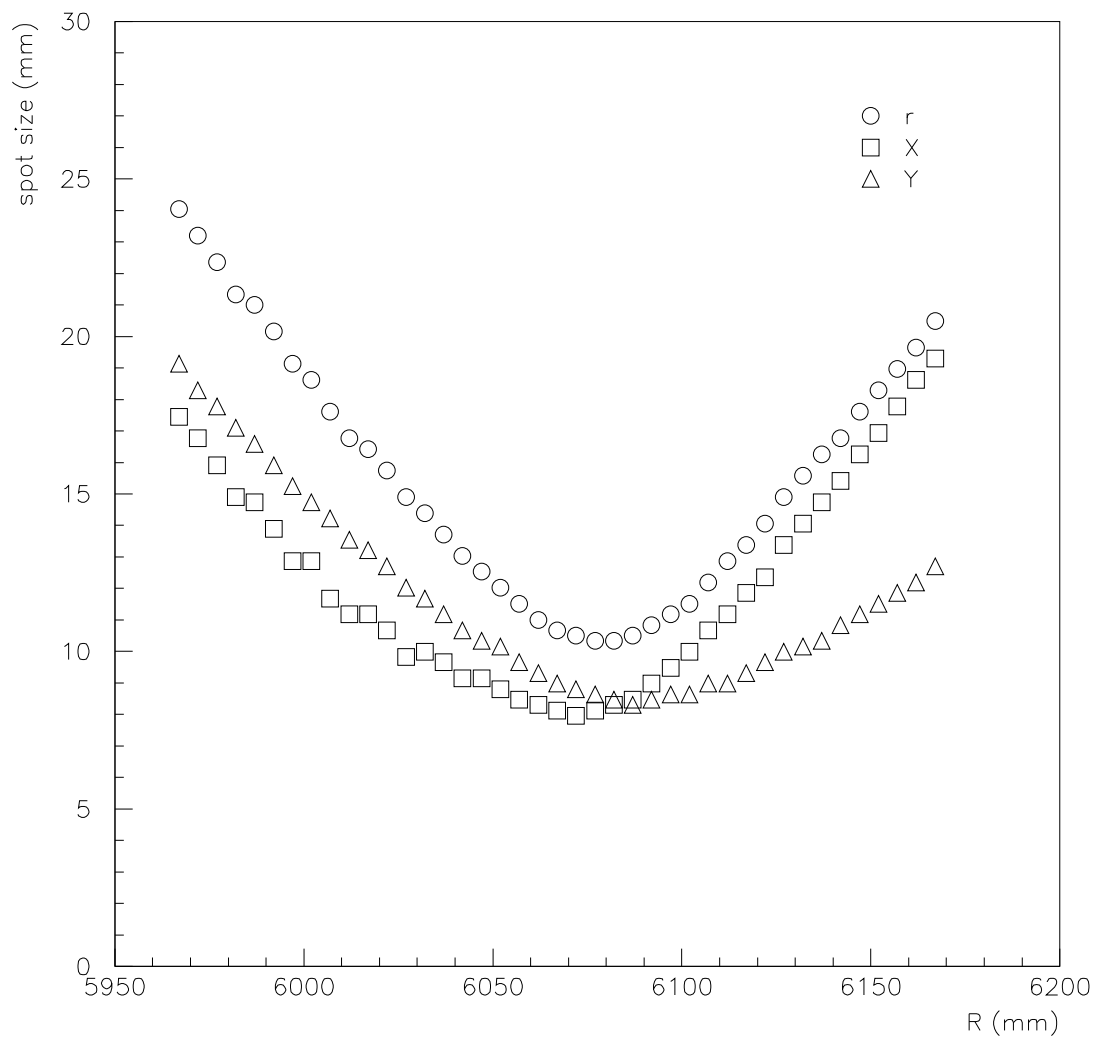

Figure 10: Typical relation between spot size and distance from a segment mirror (circles: spot sizes; squares and triangles: projected spot sizes on $\mathrm{X}$ and $\mathrm{Y}$ axes, respectively). 

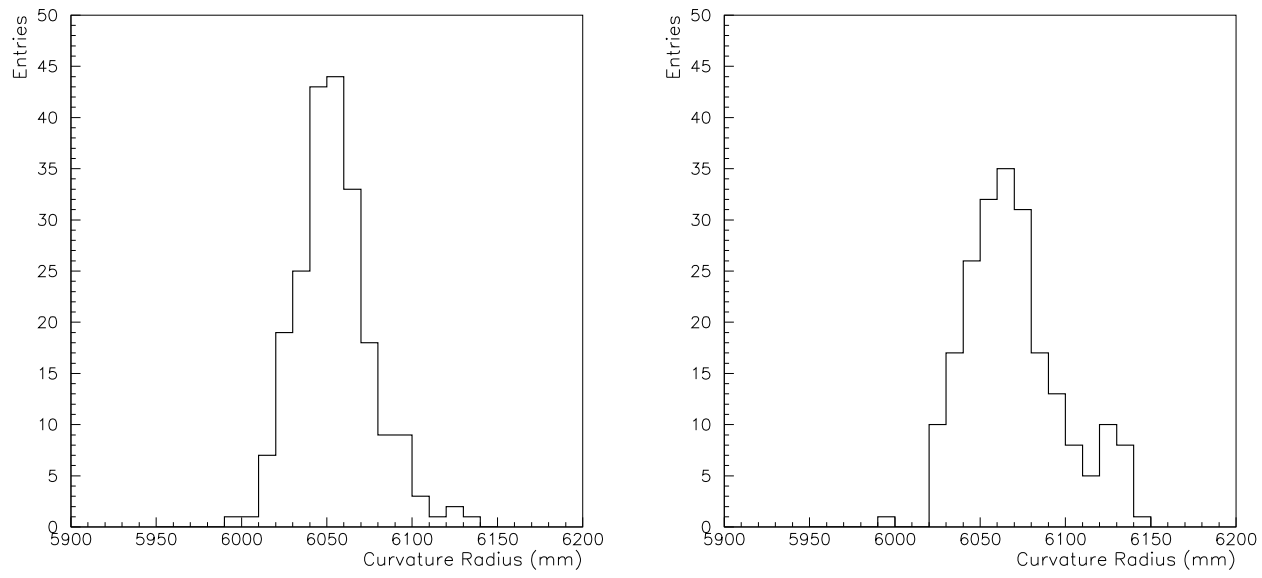

Figure 11: Distribution of the curvature radius of segment mirrors (left: BRM, right: LR). 

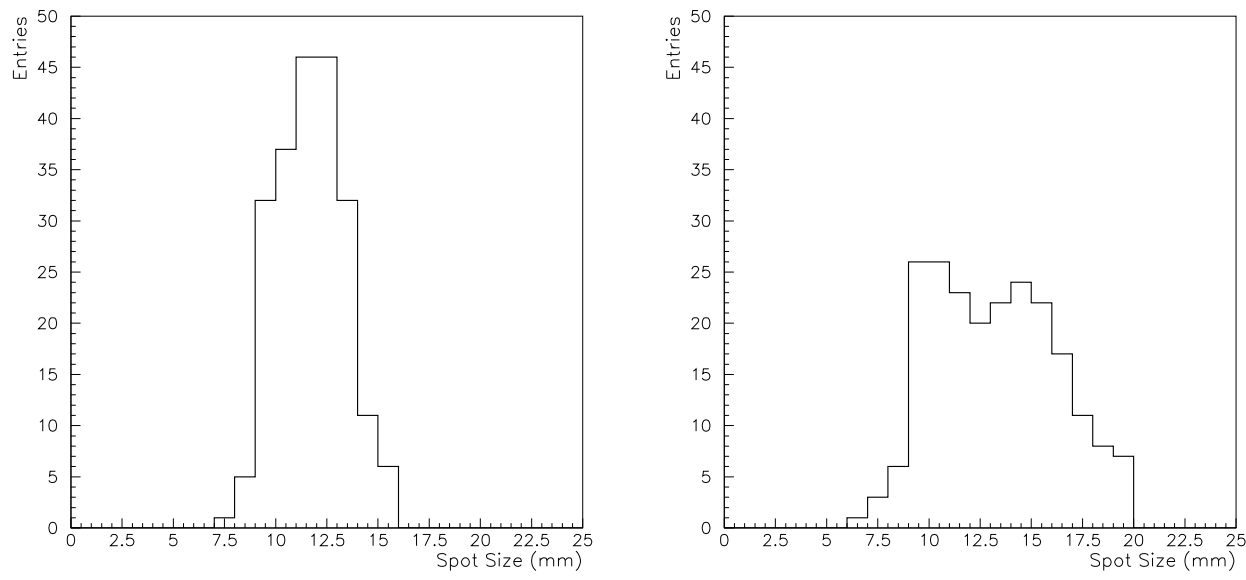

Figure 12: Distribution of the spot size of segment mirrors (left: BRM, right: LR). 


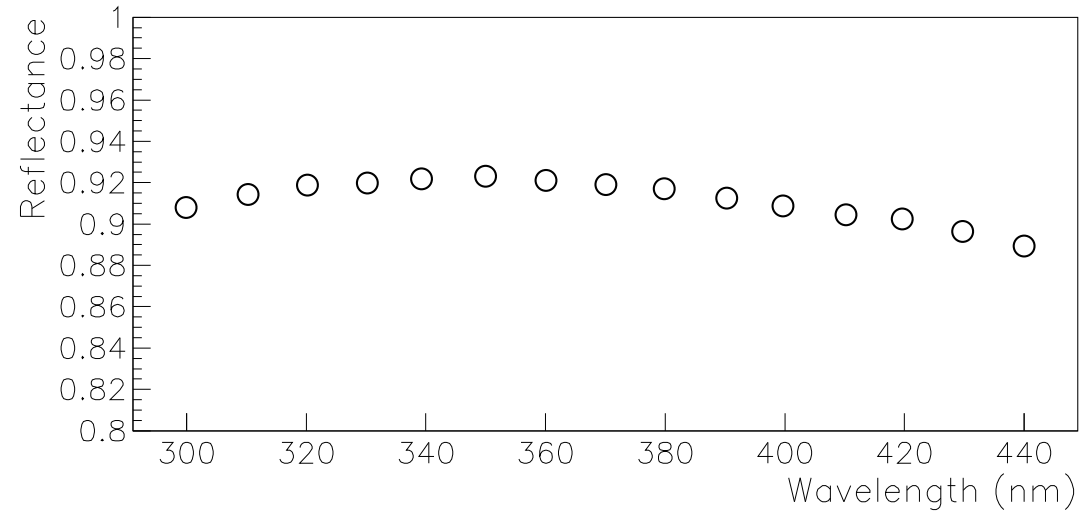

Figure 13: Typical reflectance of small flat mirror, measured by the manufacturer (systematic error and measurement error are less than 1\%). 


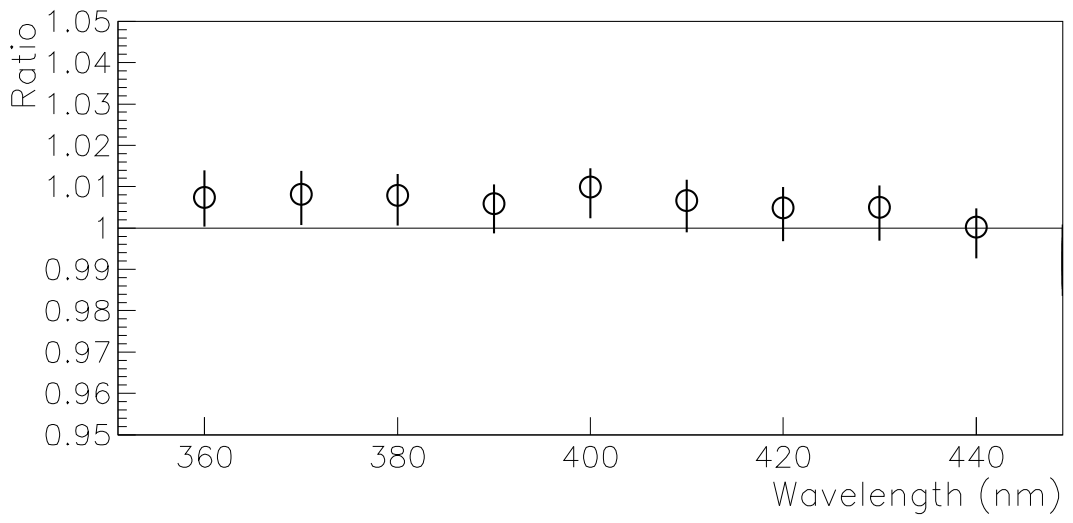

Figure 14: The comparison of mirror reflectance between the NIST measurement and our measurement (plots: our measurement value divided by the NIST one, error bars: $1 \sigma$ ). 


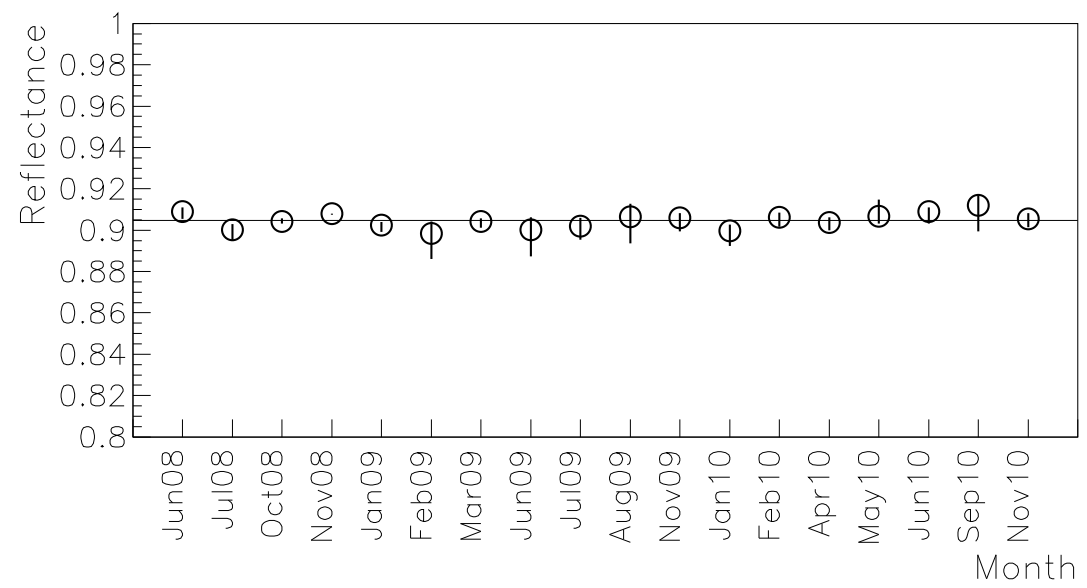

Figure 15: Variation in the reflectance of a reference mirror at $360 \mathrm{~nm}$ from June 2008 to November 2010 (plots: median value, error bars: $1 \sigma$, horizontal line: mean value of the plots). 


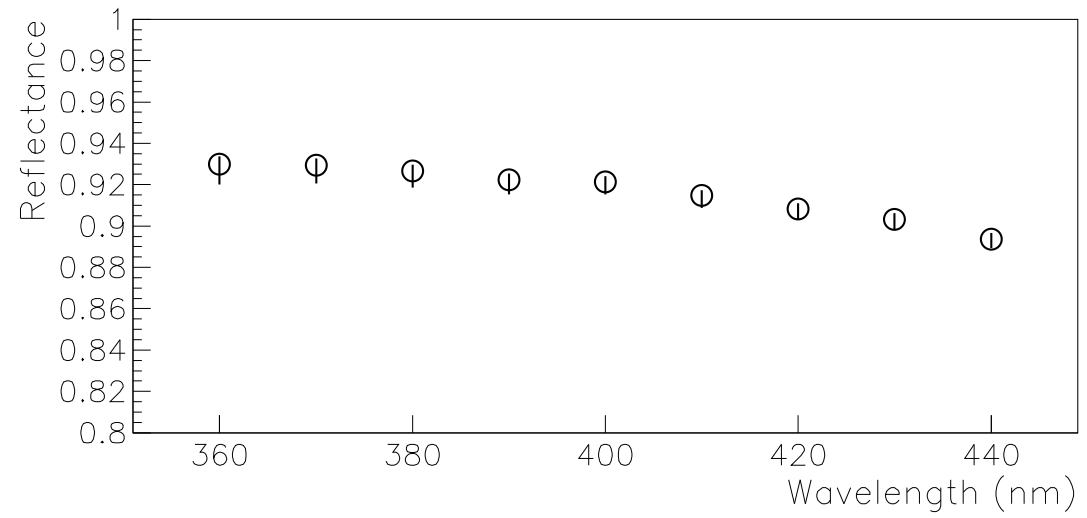

Figure 16: Wavelength dependence of the reflectance of all segment mirrors in March 2007 (plots: median value of 24 primary mirrors, error bars: $1 \sigma$ ). 


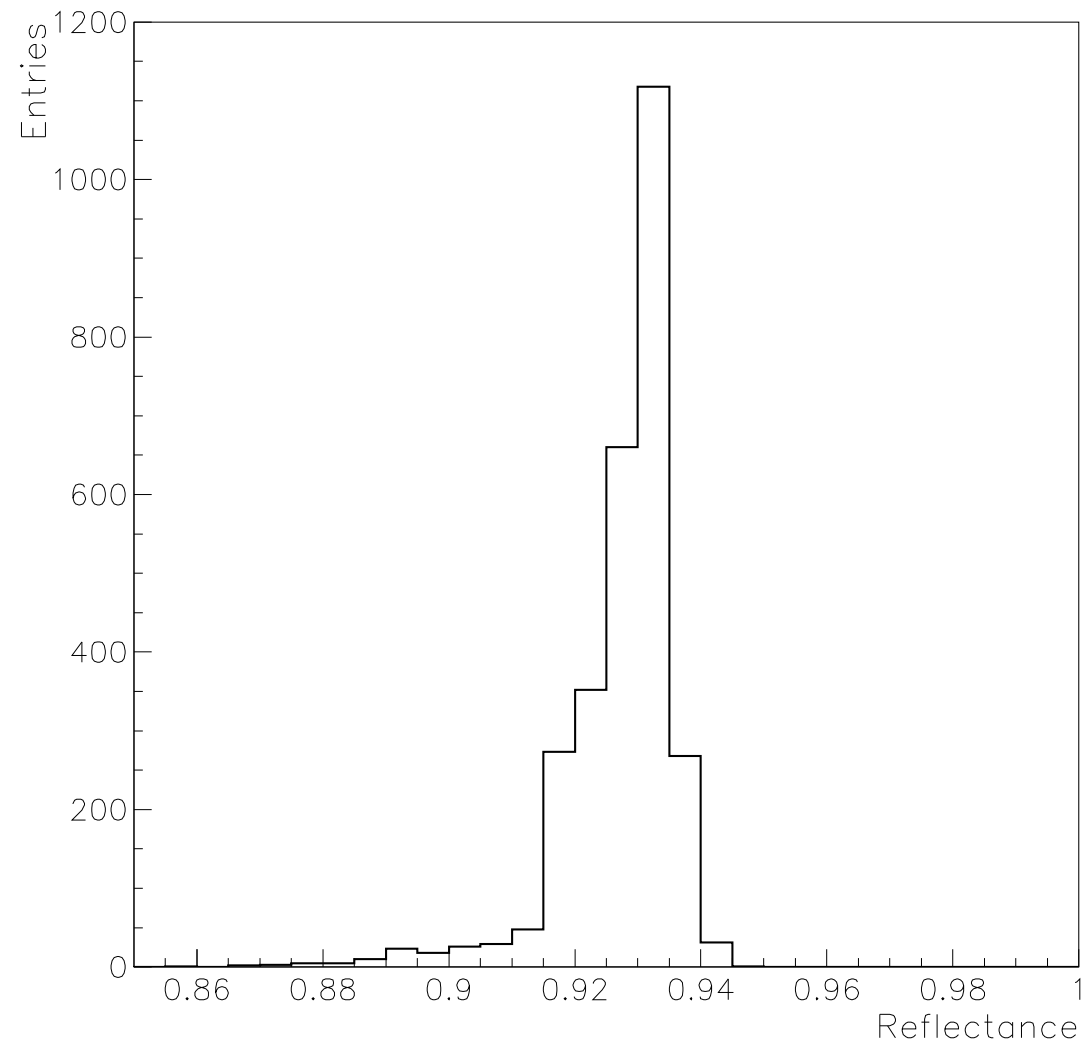

Figure 17: Distribution of mirror reflectance of 24 primary mirrors at $360 \mathrm{~nm}$ in March 2007. 


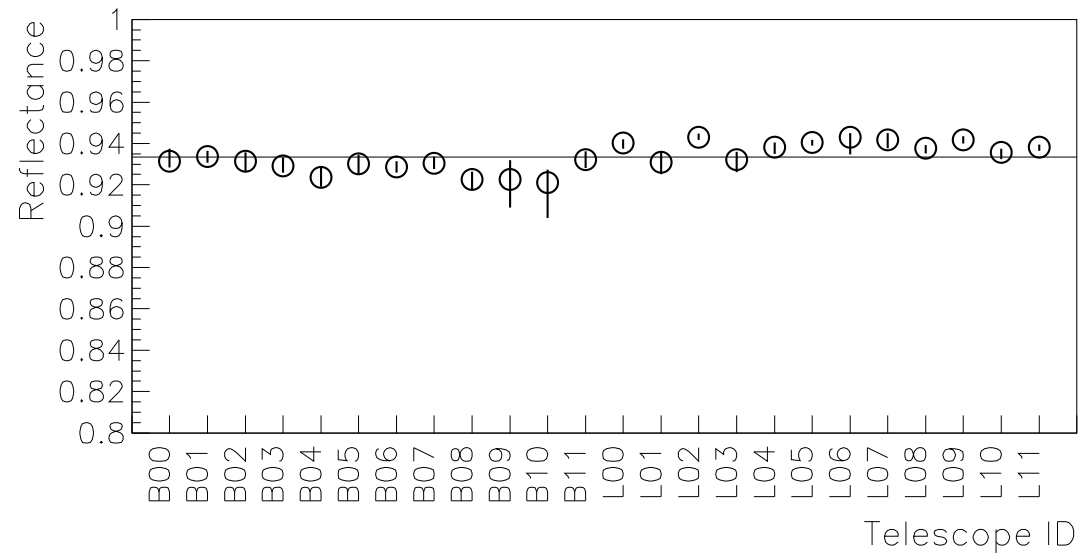

Figure 18: mirror reflectance at $360 \mathrm{~nm}$ (horizontal axis: telescope ID, B: BRM, L: LR, plots: median value of 18 segment mirrors, error bars: $1 \sigma$, horizontal line: mean value of the plots). 


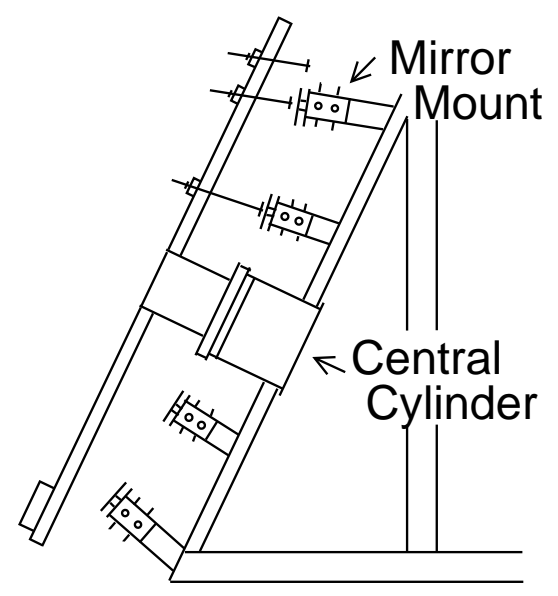

Figure 19: Schematic view of the equipment used for adjusting segment mirror mount. The equipment was mounted on the central cylinder of the mirror. 


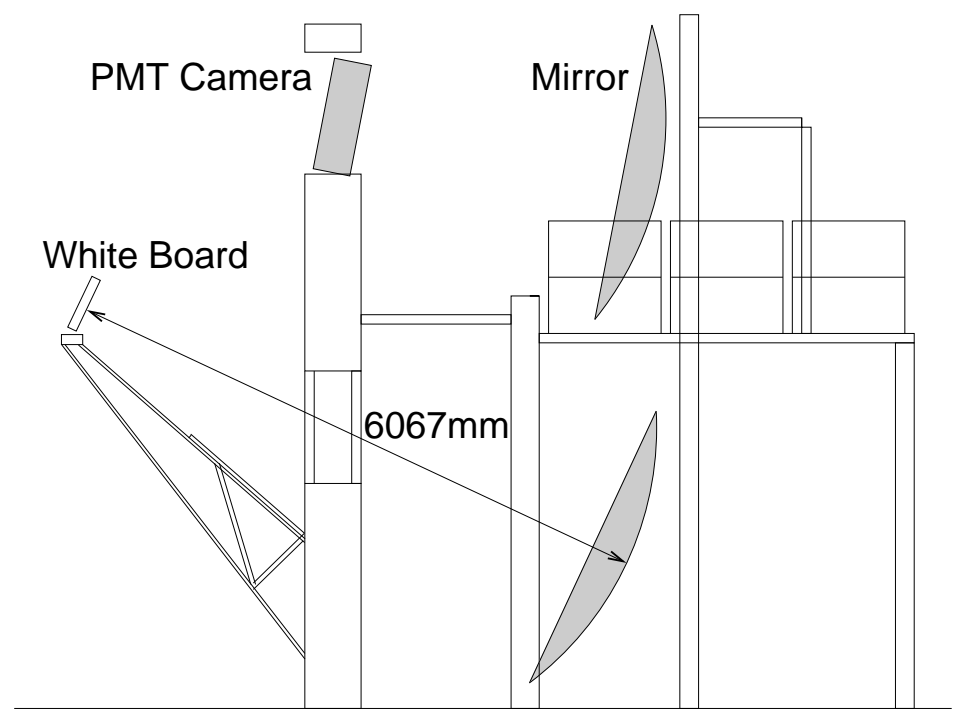

Figure 20: Schematic view of the equipment for adjusting segment mirror direction. The equipment is mounted on a camera support frame. A white board with LEDs is placed at a distance same as the mirror curvature radius of $6067 \mathrm{~mm}$ using this equipment. 


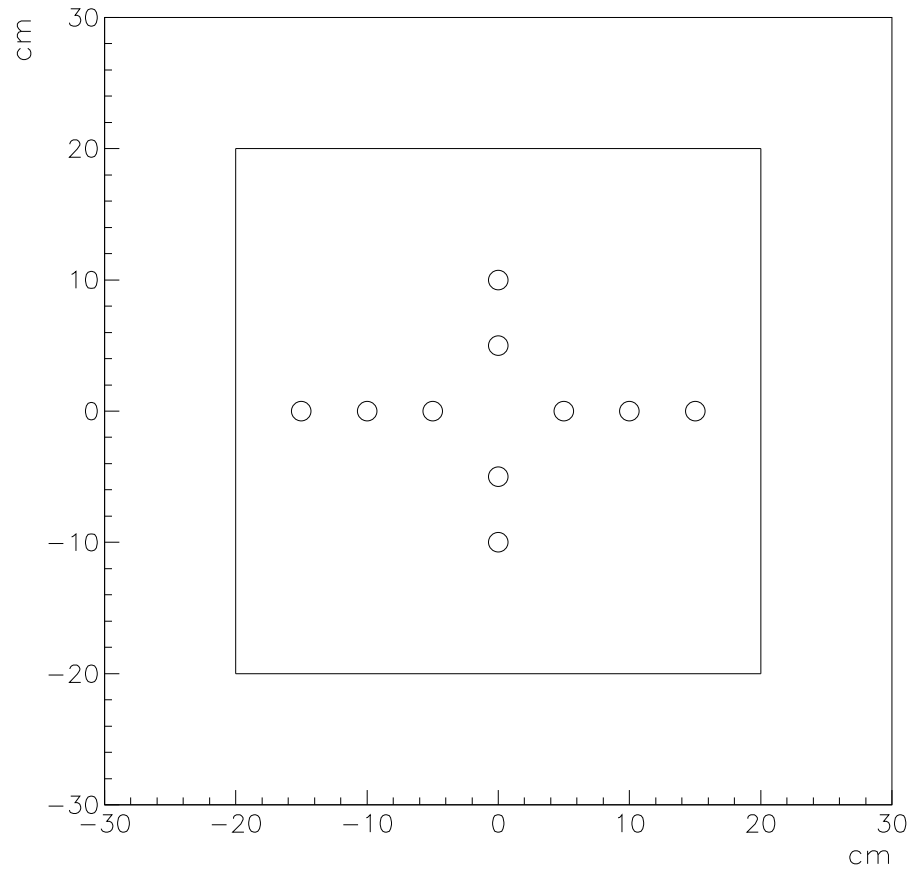

Figure 21: Schematic view of LED positions on the white board of BANANA3. 


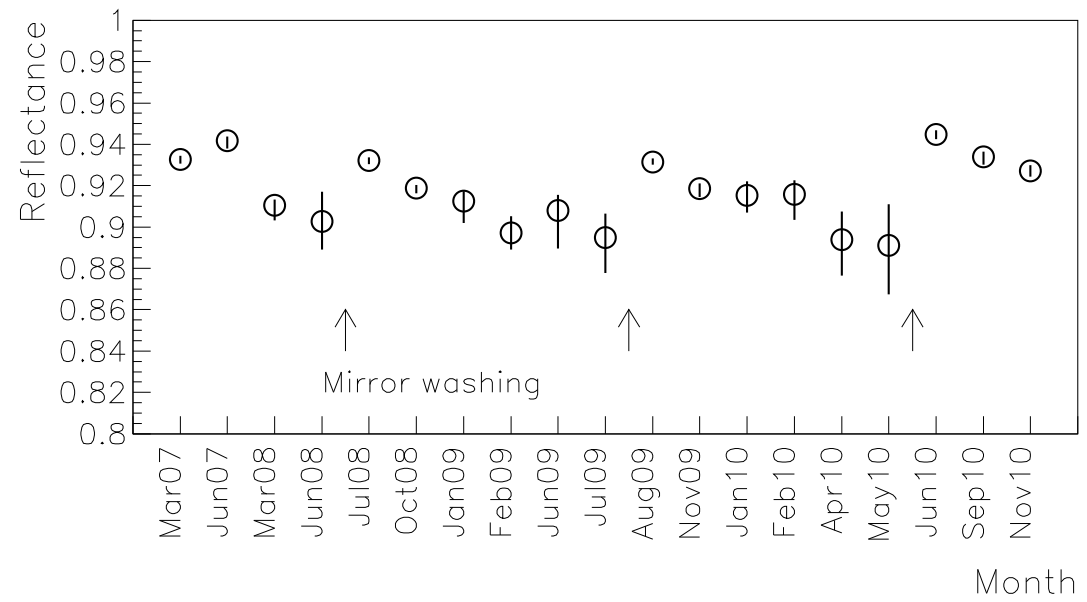

Figure 22: Variation in the mirror reflectance at $360 \mathrm{~nm}$ of a typical lower telescope LR04 (plots: median value, error bars: $1 \sigma$ ). The mirror was washed after these measurements in July 2008, August 2009, and May 2010. 


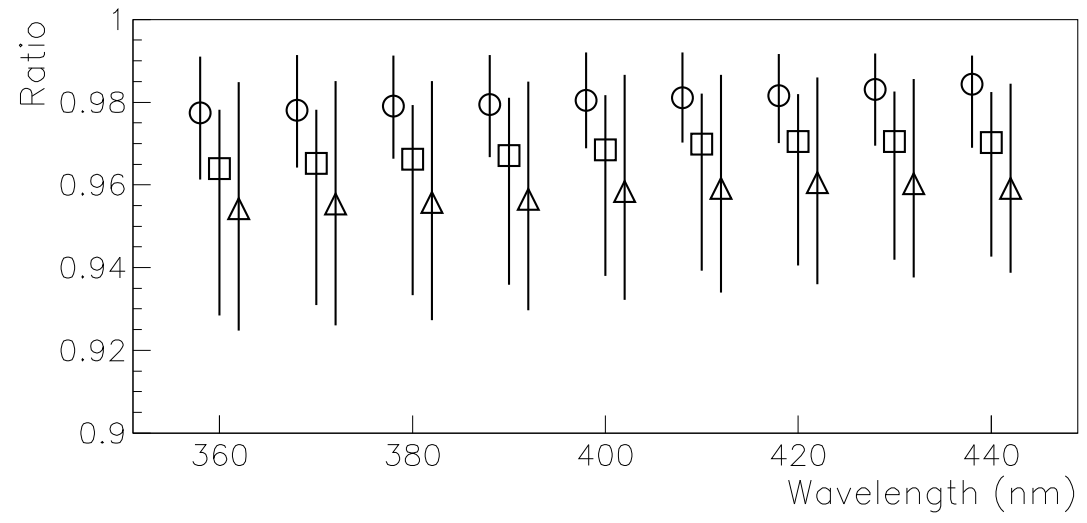

Figure 23: Differences in mirror reflectance before and after mirror washing at each wavelength (ratio: before/after, plots: median value of 24 telescopes, error bars: $1 \sigma$, circles: 2008, squares: 2009, triangles: 2010). These error bars show the non-uniformity of reflectance just before mirror washing. For visibility, the circles and triangles are slightly moved on the horizontal axis. 